\title{
Sign reversal of drag in bilayer systems with in-plane periodic potential modulation
}

\author{
Alkauskas, A.; Flensberg, Karsten; Hu, Ben Yu-Kuang; Jauho, Antti-Pekka
}

Published in:

Physical Review B Condensed Matter

Link to article, DOI:

10.1103/PhysRevB.66.201304

Publication date:

2002

Document Version

Publisher's PDF, also known as Version of record

Link back to DTU Orbit

Citation (APA):

Alkauskas, A., Flensberg, K., Hu, B. Y-K., \& Jauho, A-P. (2002). Sign reversal of drag in bilayer systems with inplane periodic potential modulation. Physical Review B Condensed Matter, 66(20), 201304.

https://doi.org/10.1103/PhysRevB.66.201304

\section{General rights}

Copyright and moral rights for the publications made accessible in the public portal are retained by the authors and/or other copyright owners and it is a condition of accessing publications that users recognise and abide by the legal requirements associated with these rights.

- Users may download and print one copy of any publication from the public portal for the purpose of private study or research.

- You may not further distribute the material or use it for any profit-making activity or commercial gain

- You may freely distribute the URL identifying the publication in the public portal 


\title{
Sign reversal of drag in bilayer systems with in-plane periodic potential modulation
}

\author{
Audrius Alkauskas, ${ }^{1,2,4}$ Karsten Flensberg, ${ }^{1}$ Ben Yu-Kuang Hu, ${ }^{3}$ and Antti-Pekka Jauho, ${ }^{4}$ \\ ${ }^{1}$ Orsted Laboratoriet, Universitetsparken 5, Kbbenhavns Universitet, DK-2100 Kbbenhavn Ф, Denmark \\ ${ }^{2}$ Physics Faculty, Vilnius University, Sauletekio 9, Vilnius 2040, Lithuania \\ ${ }^{3}$ Department of Physics, University of Akron, Akron, Ohio 44325-4001 \\ ${ }^{4}$ Mikroelektronik Centret, Danmarks Tekniske Universitet, DK-2800 Lyngby, Denmark \\ (Received 12 June 2002; revised manuscript received 28 August 2002; published 26 November 2002)
}

\begin{abstract}
We develop a theory for describing frictional drag in bilayer systems with in-plane periodic potential modulations, and use it to investigate the drag between bilayer systems in which one of the layers is modulated in one direction. At low temperatures, as the density of carriers in the modulated layer is changed, we show that it is possible for the transresistivity component in the direction of modulation to change its sign. We also give a physical explanation for this behavior.
\end{abstract}

DOI: 10.1103/PhysRevB.66.201304

Throughout the past decade there has been a great deal of experimental and theoretical activity in frictional drag in bilayer systems, following the seminal experiments by Gramila et al. ${ }^{1}$ These drag experiments involved a double quantumwell system where the layers are individually contacted by ingenious fabrication techniques. The barrier between the wells is made thick enough to suppress tunneling but thin enough to allow significant interlayer interactions. An average current density $\mathbf{j}_{1}$ is driven through layer 1 and circuit is kept open in layer 2 , so that $\mathbf{j}_{2}=0$. The interlayer interaction causes the electrons in layer 1 to drag along the electrons in layer 2, and hence a counterbalancing electric field $\mathbf{E}_{2}$ forms in layer 2 to maintain a zero net $\mathbf{j}_{2}$. The transresistivity tensor $\overleftrightarrow{\rho}_{21}$, defined by $\mathbf{E}_{2}=\overleftrightarrow{\rho}_{21} \mathbf{j} \mathbf{j}_{1}$, can be extracted experimentally and can reveal important information about the properties of the effective interlayer interactions, the individual layers and the coupled bilayer system.

Since the original work of Gramila et al., ${ }^{1}$ which was done on a closely spaced electron-electron system at low temperatures without an applied magnetic field, many variations on the theme of the original experiments have been performed. For instance, drag has been measured in electron-hole ${ }^{2}$ and hole-hole ${ }^{3}$ systems, in widely separated layers, ${ }^{4,5}$ and in the presence of a perpendicular magnetic field. ${ }^{3,6-8}$ Very recently, low-density systems have been studied to probe the suggested metal-insulator phase transition in strongly correlated disordered two-dimensional systems. ${ }^{9,10}$ Another modern trend is to examine mesoscopic effects in Coulomb drag. ${ }^{11-13}$ In general, drag without an applied $B$ field is reasonably well understood within the framework of a standard weak-interlayer coupling theory. ${ }^{14-16}$ The theory successfully accounts for several unusual features such as large enhancements in the transresistivity (up to an order of the magnitude; some intriguing discrepancies, however, do persist for the most dilute systems studied ${ }^{10}$ ) due to intralayer correlations ${ }^{17}$ and plasmon mediated scattering. ${ }^{18,19} \mathrm{On}$ the other hand, the understanding of magnetodrag (i.e., drag in the presence of a perpendicular $B$ field) in bilayer systems is less complete, and several puzzling experimental results remain unexplained. For instance, under certain circumstances, the diagonal terms in the magnetotransresistivity $\left(\rho_{21}^{x x}\right.$ and $\left.\rho_{21}^{y y}\right)$ has been observed to reverse sign when the chemical potential is changed in one layer while being kept
PACS number(s): 73.23.-b, 73.50.-h, 73.61.- $-\mathrm{r}$

fixed in the other. ${ }^{20}$ This sign reversal with changing chemical potential (which incidentally has not been observed at $B=0$ ) cannot be obtained from magnetodrag calculations using the self-consistent Born approximation, ${ }^{21,22}$ and despite recent theoretical progress, ${ }^{23}$ a fully satisfactory explanation of this phenomenon is not yet available.

In this paper, we suggest that a reversal of the sign of the transresistivity is possible at $B=0$ in bilayer systems that have periodic potential modulations in the plane of the layers. The periodic potential modulation creates minibands, and the charge carriers can evolve from electronlike to holelike behavior with a relatively small change in the density. Furthermore, for systems that are modulated in one direction, it is possible to observe anisotropic drag, implying that the electric-field response in the drag layer is in a different direction from that of the driving current. This demonstrates the important role band structure plays in determining the transresistivity of the system. ${ }^{24}$ We note that experiments on two-dimensional electron gases with strong potential modulations in one direction have already been reported in the literature, ${ }^{25}$ and hence we believe that the theory described below is amenable to experimental tests in near future.

To investigate drag in these modulated systems, we use the Kubo formalism ${ }^{15,16}$ to calculate the transconductivity tensor $\overleftrightarrow{\sigma}_{21}$, which is related to the transresistivity by $\overleftrightarrow{\rho}_{21}=$ $-\overleftrightarrow{\rho}_{22} \overleftrightarrow{\sigma}_{21} \overleftrightarrow{\rho}_{11}$ in the weak-interlayer coupling limit. In this method, the transconductivity is expressed as a currentcurrent correlation function, which can be calculated with standard perturbation-theory techniques.

The Hamiltonian of the system is $\hat{H}=\sum_{i=1,2} \hat{H}_{i}+\hat{H}_{12}$, where $\hat{H}_{i}$ is the Hamiltonian of layer $i$ and $\hat{H}_{12}$ is the interlayer interaction term. We assume $\hat{H}_{21}$ is due to Coulomb interactions, so that $\hat{H}_{12}=\mathcal{A}^{-1} \sum \hat{n}_{1}(\mathbf{q}) \hat{n}_{2}(-\mathbf{q}) V_{12}(\mathbf{q})$, where $\hat{n}_{i}(\mathbf{q})$ and $V_{12}(\mathbf{q})$ are the Fourier transforms of the density operator and the interlayer Coulomb interaction, respectively.

We define, within the Matsubara formalism, $\vec{\Delta}$ to be the correlation function ${ }^{26}$

$$
\begin{aligned}
\vec{\Delta}\left(\mathbf{q}, \mathbf{q}^{\prime} ; i \omega_{n}, i \omega_{n}^{\prime}\right)= & -\int_{0}^{\beta} d \tau \int_{0}^{\beta} d \tau^{\prime} e^{i \omega_{n} \tau} e^{-i \omega_{n}^{\prime} \tau^{\prime}} \\
& \times\left\langle T_{\tau} \hat{\mathbf{J}}(0) \hat{n}(\mathbf{q}, \tau) \hat{n}\left(-\mathbf{q}^{\prime}, \tau^{\prime}\right)\right\rangle_{0} .
\end{aligned}
$$


For systems that have a periodic potential modulation with reciprocal vectors $\mathbf{G}$, only $\mathbf{q}-\mathbf{q}^{\prime}=\mathbf{G}$ terms are nonzero. Expanding in powers of $V_{12}$, the first nonvanishing term for $\sigma_{21}$ in the dc limit is the second-order term. We obtain

$$
\begin{aligned}
\sigma_{21}^{\delta \gamma}= & \frac{e^{2}}{h \mathcal{A}} \sum_{\mathbf{q}} \sum_{\mathbf{G}_{\mathbf{1}} \mathbf{G}_{\mathbf{2}}} V_{12}(\mathbf{q}) V_{12}\left(-\mathbf{q}+\mathbf{G}_{\mathbf{1}}\right) \delta_{\mathbf{G}_{\mathbf{1}} \mathbf{G}_{\mathbf{2}}} \\
& \times \int_{0}^{\infty} \frac{d \omega}{2 \pi} \Delta_{2}^{\delta}\left(\mathbf{q}, \mathbf{q}+\mathbf{G}_{\mathbf{2}} ; \omega+i 0^{+}, \omega-i 0^{+}\right) \\
& \times \Delta_{1}^{\gamma}\left(\mathbf{q}, \mathbf{q}+\mathbf{G}_{\mathbf{1}} ; \omega+i 0^{+}, \omega-i 0^{+}\right)\left[-\partial_{\omega} n_{B}(\omega)\right] .
\end{aligned}
$$

Evaluation of $\vec{\Delta}\left(\mathbf{q}, \mathbf{q}+\mathbf{G} ; \omega+i 0^{+}, \omega-i 0^{+}\right)$is analogous to Ref. 16. In this paper, we assume the system is in the weak scattering limit, $\ell \gg d, a$ ( $\ell$ is the impurity mean-free path, $d$ is the layer separation, and $a$ is the modulation period) ${ }^{27}$ allowing us to ignore vertex corrections at the charge vertices. Then, one obtains

$$
\begin{aligned}
\vec{\Delta}(\mathbf{q}, \mathbf{q} & \left.+\mathbf{G}, \omega+i 0^{+}, \omega-i 0^{+}\right) \\
= & \frac{4 \pi}{\mathcal{A}} \sum_{\mathbf{k} n n^{\prime}}\left[\mathbf{v}_{n^{\prime}} \mathbf{k}+\mathbf{q}_{\mathrm{tr}, n^{\prime}}(\mathbf{k}+\mathbf{q})-\mathbf{v}_{n \mathbf{k}} \tau_{\mathrm{tr}, n}(\mathbf{k})\right] \\
& \times\left[\left(n_{F}\left(\varepsilon_{n \mathbf{k}}\right)-n_{F}\left(\varepsilon_{n \mathbf{k}}-\omega\right)\right] \delta\left(\varepsilon_{n \mathbf{k}^{\prime}}-\varepsilon_{n^{\prime}} \mathbf{k}+\mathbf{q}-\omega\right)\right. \\
& \times \eta\left(\mathbf{k}+\mathbf{q} n^{\prime}, \mathbf{k} n ; \mathbf{q}\right) \eta\left(\mathbf{k} n, \mathbf{k}+\mathbf{q} n^{\prime} ;-\mathbf{q}-\mathbf{G}\right) .
\end{aligned}
$$

Here, $\mathbf{v}_{n \mathbf{k}}$ is the band velocity, $\varepsilon$ is the energy, $n_{F}(\varepsilon)$ $=\{\exp [\beta(\varepsilon-\mu)]+1\}^{-1}(\mu$ is the chemical potential $), \tau_{\text {tr }}$ is the transport time, $\eta\left(\mathbf{k}^{\prime} n^{\prime}, \mathbf{k} n ; \mathbf{q}\right)=\left\langle\mathbf{k}^{\prime} n^{\prime}|\exp (-i \mathbf{q} \cdot \mathbf{r})| \mathbf{k} n\right\rangle$ $\left(n, n^{\prime}\right.$ are the band indices). The $\vec{\sigma}_{21}$ obtained using Eq. (2) and the weak scattering result Eq. (3) can, with the approximation $\eta=1$, also be derived from the semiclassical Boltzmann equation. ${ }^{19}$

A complete calculation of drag, using Eq. (3) in Eq. (2), is an arduous task, requiring a numerical evaluation of the band structure(s) of the layers, calculation of the matrix elements $\eta\left(\mathbf{k}^{\prime} n^{\prime}, \mathbf{k} n ; \mathbf{q}\right)$, and summation of different bands $n$, and reciprocal lattice vectors $\mathbf{G}$. For incommensurate lattices one always has $\mathbf{G}_{\mathbf{1}}=0=\mathbf{G}_{\mathbf{2}}$, and in the remaining part of the paper we assume this to be the case. The other technical steps do not pose conceptual difficulties, and in the present context we find it appropriate to consider simplified systems where to a certain extent analytic progress can be made, and for which the physics is transparent.

The central issue of this paper is the possible sign reversal of the drag. We demonstrate this first for a one-dimensional model ${ }^{28}$ neglecting interband processes and the momentum dependence of the transport relaxation time. For this case, correlation function (3) becomes $\Delta=\tau_{\mathrm{tr}} F(q, \omega)$, where

$$
\begin{aligned}
F(q, \omega)= & -\frac{2 \pi}{\mathcal{L}} \sum_{k}\left(v_{k}-v_{k+q}\right) \\
& \times\left[n_{F}\left(\varepsilon_{k}\right)-n_{F}\left(\varepsilon_{k+q}\right)\right] \delta\left(\varepsilon_{k}-\varepsilon_{k+q}-\omega\right) \\
= & \sum_{k_{i}} \operatorname{sign}\left(v_{k_{i}}-v_{k_{i}+q}\right)\left[n_{F}\left(\varepsilon_{k_{i}}\right)-n_{F}\left(\varepsilon_{k_{i}+q}\right)\right],
\end{aligned}
$$

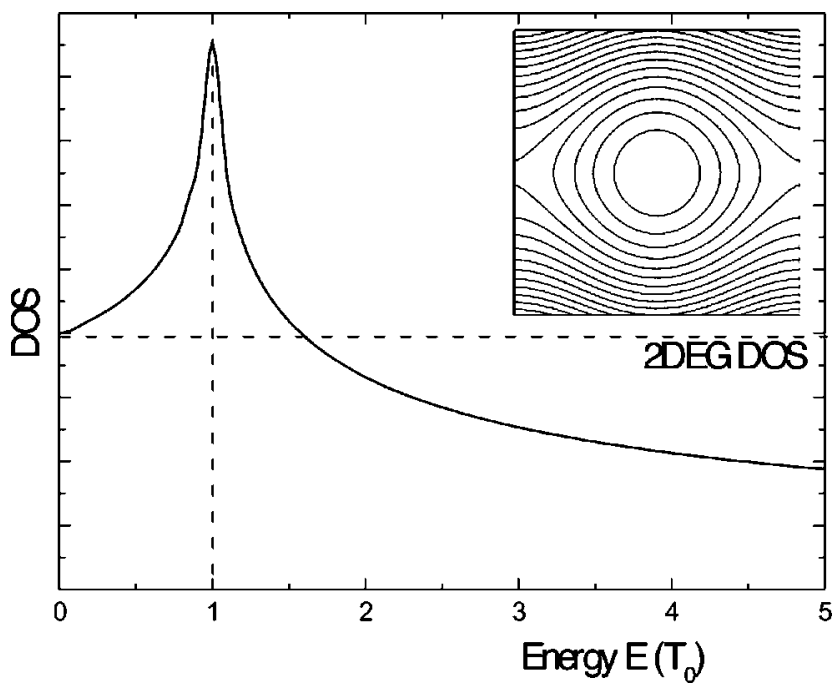

FIG. 1. The density of states for a two-dimensional system with periodical modulation in one direction. The inset shows the constant energy surfaces for the dispersion relation used in this work. The energy is in units of $k_{B} T_{0}=2 \hbar^{2} /\left(m_{x} a^{2}\right)$.

where $k_{i}$ are the solutions of $\varepsilon_{k_{i}}-\varepsilon_{k_{i}+q}-\omega=0$. For illustrative purposes, we consider a cosine band, $\varepsilon_{k}=$ $-\hbar^{2} /\left(m a^{2}\right) \cos k a$, for which there are two (or no) solutions, and one finds

$$
\begin{aligned}
F(q, \omega)= & \operatorname{sign}\left(v_{2}-v_{1}\right)\left[n_{F}\left(-\varepsilon_{2}\right)-n_{F}\left(\varepsilon_{1}\right)-n_{F}\left(-\varepsilon_{1}\right)\right. \\
& \left.-n_{F}\left(\varepsilon_{2}\right)\right] .
\end{aligned}
$$

At half-filling the chemical potential $\mu$ vanishes, and making use of $n_{F, \mu}(-\varepsilon)=1-n_{F,-\mu}(\varepsilon)$, it is easy to see that the result (5) is an odd function of $\mu$. Thus, in an experiment where one of the subsystems is kept unchanged while in the other the chemical potential is moved through half-filling, the drag will change sign. While the above discussion is an important demonstration of principle, it is necessary to also consider periodically modulated two-dimensional electron gases, which are the most commonly studied systems in this context.

A system which has an identical periodic modulation in both $x$ and $y$ directions is characterized by particle-hole symmetry, and it seems natural that the drag passes through zero when the two carrier species are matched. The experimentally most relevant systems are those, however, where the modulation is only in one direction ${ }^{25}$ (the strongest modulations have been achieved for these systems), and hence we choose the model system as follows: (1) There is a single band (the dispersion law and corresponding density of states are illustrated in Fig. 1) with a tight-binding dispersion relation ${ }^{29} \quad \varepsilon\left(k_{x}, k_{y}\right)=\hbar^{2}\left[1-\cos \left(k_{x} a\right)\right] /\left(m_{x} a^{2}\right)+\hbar^{2} k_{y}^{2} /$ $\left(2 m_{y}\right)$, and hence the velocity components are $v_{x}\left(k_{x}\right)$ $=\hbar \sin \left(k_{x} a\right) /\left(m_{x} a\right)$ and $v_{y}\left(k_{y}\right)=\hbar k_{y} / m_{y}$. (2) $\tau_{\text {tr }}$ is $\mathbf{k}$ independent. (3) The interlayer interaction $V_{12}(q)$ is significant only for small $q{ }^{30}$

At low temperatures, it would appear permissible to expand in $\omega$, because $\partial n_{B}(\omega) / \partial \omega$ in the integrand cuts off the higher $\omega$ contributions. Following this procedure yields an analytic expression for $F$, and the resulting drag resistivity 


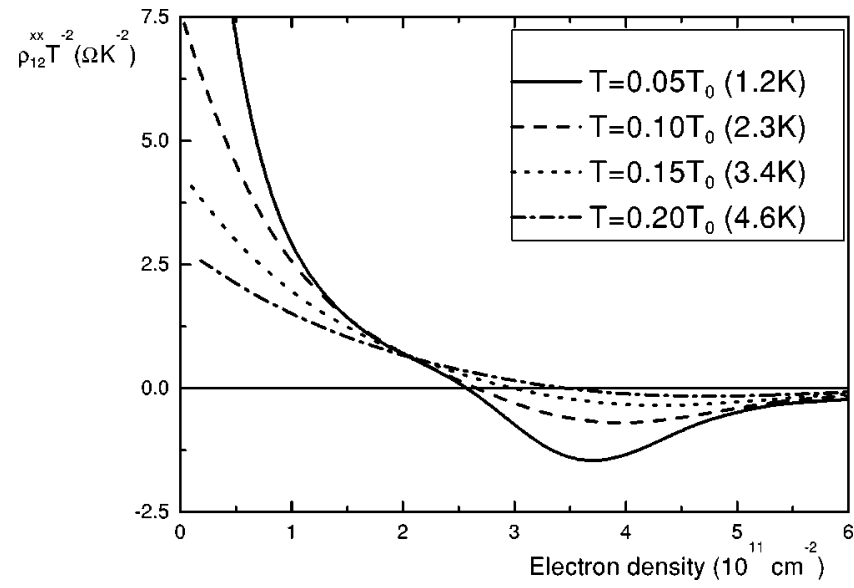

FIG. 2. The calculated transresistivity $\rho_{D}^{x x}$, as a function of the density for four different temperatures. Superlattice period $a=140 \AA$, and the layer separation is $280 \AA$.

obeys the familiar $T^{2}$ dependence known from unmodulated two-dimensional systems. ${ }^{1,14}$ In this scheme, the transresisitivity diverges when $\mu=2 \hbar^{2} /\left(m_{x} a^{2}\right)$ with the opposite sign from the low density $\rho^{x x}$. This divergence, which is related to the divergence in the density of states at this energy (see Fig. 1), is unphysical because it only occurs in the experimentally unreachable $T=0$ limit. Nevertheless, it is interesting to observe that this approximation leads to a change of sign in the transconductance, and we should expect this behavior to be most prominent when $\mu=2 \hbar^{2} /\left(m_{x} a^{2}\right)$.

To cure this spurious divergence one must avoid expansions, and perform a numerical evaluation. As a starting point we have found it convenient to use

$$
\begin{aligned}
F^{x}(\mathbf{q}, \omega)= & \frac{m_{y}}{(2 \pi)^{2} \hbar q_{y} m_{x} a} \int_{-\pi / a+q_{x} / 2}^{\pi / a-q_{x} / 2} d k_{x}\left[\sin \left(k_{x}-q_{x} / 2\right) a\right. \\
& \left.-\sin \left(k_{x}+q_{x} / 2\right) a\right]\left\{n_{F}\left[k_{x}-q_{x} / 2, k_{y 0}\left(k_{x}\right)-q_{y} / 2\right]\right. \\
& \left.-n_{F}\left[k_{x}+q_{x} / 2, k_{y 0}\left(k_{x}\right)+q_{y} / 2\right]\right\},
\end{aligned}
$$

where

$$
\begin{aligned}
k_{y 0}\left(k_{x}\right)= & \frac{m_{y}}{\hbar^{2} q_{y}}\left[\hbar \omega+\frac{\hbar^{2}}{m_{x} a^{2}}\left[\cos \left(k_{x}+q_{x} / 2\right) a\right.\right. \\
& \left.\left.-\cos \left(k_{x}-q_{x} / 2\right) a\right]\right] .
\end{aligned}
$$

While the Kubo formula gives the transconductivity, it is often most convenient to express the results in terms of transresisistivity (this is the object usually recorded in experiments) $\stackrel{\leftrightarrow}{\rho}_{21}$, whose components are given by

$$
\rho_{21}^{x x}=-\frac{\sigma_{21}^{x x}}{\sigma_{11}^{x x} \sigma_{22}^{x x}-\sigma_{12}^{x x} \sigma_{21}^{x x}} \simeq-\frac{\sigma_{21}^{x x}}{\sigma_{11}^{x x} \sigma_{22}^{x x}},
$$

and analogously for the $y y$ component. The transresisivity tensor has the additional advantage that does not involve the transport relaxation times for the individual layers, as long as

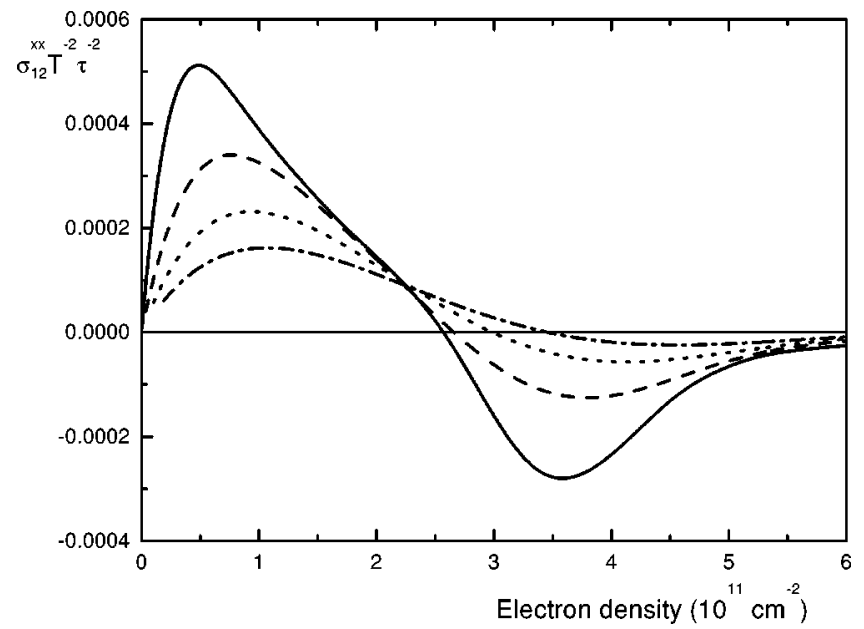

FIG. 3. The normalized transconductivity $\sigma_{D}^{x x}$, as a function of the density for the same temperatures as in Fig. 1.

these are momentum independent. The computed transresisitivity is shown in Fig. 2 for four different temperatures. The most important feature is that the drag indeed changes sign; the effect is most prominent for low temperatures, and densities close to a fully occupied band. ${ }^{31}$ In Figs. 3 and 4 we compare the $x x$ and $y y$ components of the computed transconductances (the $x x$ component was used in calculating the results of Fig. 2). We observe that the sign change does not take place for $\sigma_{12}^{y y}$, nevertheless an interesting double-peak structure emerges.

An analysis of the several assumptions made in our calculations is now in place. We have assumed that the system only has one band. Clearly, this assumption breaks down when the density so large that Fermi energy significantly exceeds $2 \hbar^{2} /\left(2 m_{x} a^{2}\right)$, because the carriers will start to occupy higher bands. We also have assumed that temperature is low enough that the inelastic mean-free path $\ell_{\text {in }}$ is much longer than period of the potential modulation, $a$. For finite $\ell_{\text {in }}$ the system is roughly divided into coherent regions of order $\ell_{\text {in }}^{2}$. If $\ell_{\text {in }} \lesssim a$, the electrons do not coherently feel the

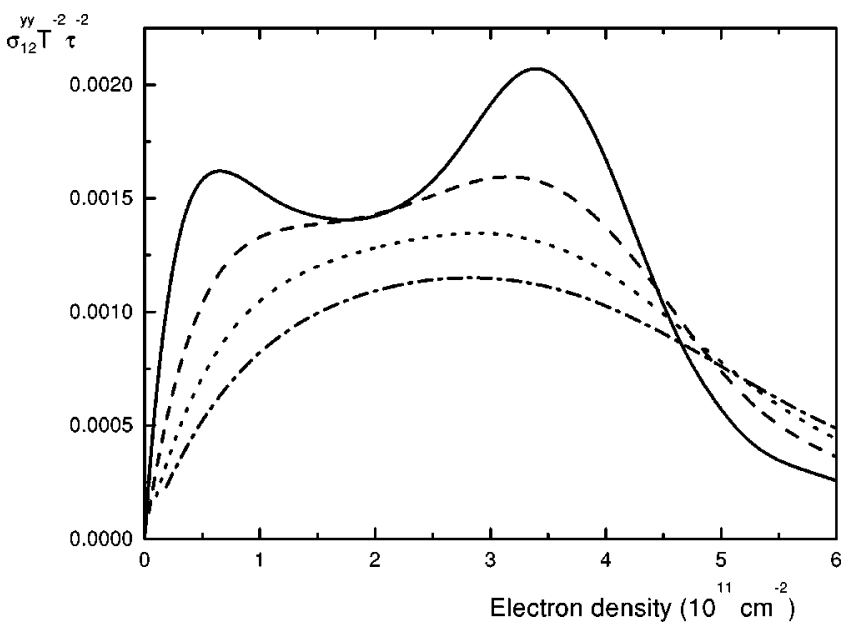

FIG. 4. The normalized transconductivity $\sigma_{D}^{y y}$, as a function of the density for four different temperatures. 
periodic potential, and the drag characteristics will be given by an average of the drag over the density fluctuation caused by the potential modulation. Since the system as a whole acts like a (nearly) uniform system in this case, effects described in this paper will not be observable at temperatures for which $\ell_{\text {in }} \lesssim a$.

To summarize, we have developed a theory for drag in bilayer systems where there is a periodic potential modulation. We have calculated the drag for the case where there is potential modulation in one direction in one of the two layers. For the studied system, at low temperatures, the transre- sistivity changes sign as the density is increased. ${ }^{31}$ Experimentally, it may be possible to fabricate the system investigated here by overgrowing a pair of quantum wells over a cleaved edge, ${ }^{25}$ or using lithographic techniques. ${ }^{32}$

A.A. acknowledges a NORFA grant, which was crucial for this study. B.Y.K.H. was supported by DOE Grant No. DE-G02001ER45948, Research Corporation Grant No. CC5168, and by the University of Akron. We are pleased to acknowledge collaboration with Niels Asger Mortensen and Henrik Smith during the early stages of this project.
${ }^{1}$ T.J. Gramila, J.P. Eisenstein, A.H. MacDonald, L.N. Pfeiffer, and K.W. West, Phys. Rev. Lett. 66, 1216 (1991).

${ }^{2}$ U. Sivan, P.M. Solomon, and H. Shtrikman, Phys. Rev. Lett. 68, 1196 (1992).

${ }^{3}$ C. Jörger, S.J. Cheng, H. Rubel, W. Dietsche, R. Gerhardts, P. Specht, K. Eberl, and K. von Klitzing, Phys. Rev. B 62, 1572 (2000).

${ }^{4}$ T.J. Gramila, J.P. Eisenstein, A.H. MacDonald, L.N. Pfeiffer, and K.W. West, Phys. Rev. B 47, 12957 (1993).

${ }^{5}$ T.J. Gramila, J.P. Eisenstein, A.H. MacDonald, L.N. Pfeiffer, and K.W. West, Physica B 197, 442 (1994).

${ }^{6}$ N.P.R. Hill, J.T. Nicholls, E.H. Linfield, M. Pepper, D.A. Ritchie, A.R. Hamilton, G.A.C. Jones, J. Phys.: Condens. Matter 8, L557 (1996).

${ }^{7}$ H. Rubel, A. Fisher, W. Dietsche, K. von Klitzing, and K. Eberl, Phys. Rev. Lett. 78, 1763 (1997).

${ }^{8}$ M.P. Lilly, J.P. Eisenstein, L.N. Preiffer, and K.W. West, Phys. Rev. Lett. 80, 1714 (1998).

${ }^{9}$ R. Pillarisetty, Hwayong Noh, D.C. Tsui, E.P. De Poortere, E. Tutuc, and M. Shayegan, Phys. Rev. Lett. 89, 016805 (2002).

${ }^{10}$ E.H. Hwang and S. Das Sarma, cond-mat/0202249 (unpublished).

${ }^{11}$ B.N. Narozhny and I.L. Aleiner, Phys. Rev. Lett. 84, 5383 (2000).

${ }^{12}$ N.A. Mortensen, K. Flensberg, and A.P. Jauho, Phys. Rev. Lett. 86, 1841 (2001).

${ }^{13}$ N.A. Mortensen, K. Flensberg, and A.P. Jauho, Phys. Rev. B 65, 085317 (2002).

${ }^{14}$ A.-P. Jauho and H. Smith, Phys. Rev. B 47, 4420 (1993).

${ }^{15}$ A. Kamenev and Y. Oreg, Phys. Rev. B 52, 7516 (1995).

${ }^{16}$ K. Flensberg, B.Y.-K. Hu, A.-P. Jauho, and J. Kinaret, Phys. Rev. B 52, 14761 (1995).

${ }^{17}$ L. Świerkowski, J. Szymański, and Z.W. Gortel, Phys. Rev. Lett. 74, 3245 (1995).

${ }^{18}$ K. Flensberg and B.Y.-K. Hu, Phys. Rev. Lett. 73, 3572 (1994).

${ }^{19}$ K. Flensberg and B.Y.-K. Hu, Phys. Rev. B 52, 14796 (1995).

${ }^{20}$ J.G.S. Lok, S. Kraus, M. Pohlt, W. Dietsche, K. von Klitzing, W. Wegscheider, and M. Bichler, Phys. Rev. B 63, 041305(R) (2001).
${ }^{21}$ M.C. Bønsager, K. Flensberg, B.Y.-K. Hu, and A.-P. Jauho, Phys. Rev. Lett. 77, 1366 (1996).

${ }^{22}$ M.C. Bønsager, K. Flensberg, B.Y.-K. Hu, and A.-P. Jauho, Phys. Rev. B 56, 10314 (1997).

${ }^{23}$ F. von Oppen, S.H. Simon, and A. Stern, Phys. Rev. Lett. 87, 106803 (2001).

${ }^{24}$ Miniband effects have recently been observed by C. Albrecht, J.H. Smet, D. Weiss, K. von Klitzing, R. Hennig, M. Langenbuch, M. Suhrke, U. Rossler, V. Umansky, and H. Schweizer, Phys. Rev. Lett. 83, 2234 (1999).

${ }^{25}$ R.A. Deutschmann, W. Wegscheider, M. Rother, M. Bichler, G. Abstreiter, C. Albrecht, and J.H. Smet, Phys. Rev. Lett. 86, 1857 (2001).

${ }^{26}$ Note that this definition is different from the $\Delta$ used in Ref. 16. The $\Delta$ defined in Ref. 16 is appropiate only for homogenous systems.

${ }^{27}$ Strong scattering (diffusive) effects are negligible at $B=0$, see, e. g., L. Zheng and A.H. MacDonald, Phys. Rev. B 48, 8203 (1993).

${ }^{28}$ Importantly, significant progress towards fabricating modulated one-dimensional nanowires has recently been achieved by M.S. Gudiksen, L.J. Lauhon, J. Wang, D.C. Smith, and C.M. Lieber, Nature (London) 415, 617 (2002).

${ }^{29}$ This energy spectrum underlies the magnetoresistance oscillations discovered by D. Weiss, K. von Klitzing, K. Ploog, and G. Weimann, Europhys. Lett. 8, 179 (1989).

${ }^{30}$ In our calculations we use the statically screened Coulomb interaction, $V(q)=\left(2 \pi e^{2} / \kappa\right) q /\left[2 q_{\mathrm{TF}, 1} q_{\mathrm{TF}, 2} \sinh q d+q\left(q+q_{\mathrm{TF}, 1}\right.\right.$ $\left.\left.+q_{\mathrm{TF}, 2}\right) \exp q d\right]$, where $q_{\mathrm{TF}, i}$ are the Thomas-Fermi screening wave numbers for the two layers. For the modulated layer the screening wave number has a slight temperature and density dependence, which, however, has a rather small effect for the calculated drag.

${ }^{31}$ It can be shown that a necessary condition for the sign change of $\sigma_{2} 1^{i i}$ is that $\partial\left[\tau_{\mathrm{tr}}(\mathbf{k}) v_{i}(\mathbf{k})\right] / \partial k_{i}$ vanishes when integrated over the Fermi surface. The sign-change is thus not a special property of the cosine-band.

${ }^{32}$ T. J. Gramila (private communication). 